\title{
Gut microbiota metabolites as integral mediators in cardiovascular diseases (Review)
}

\author{
YING ZHU ${ }^{1,2^{*}}$, XIAORONG SHUI ${ }^{3 *}$, ZHENG LIANG $^{2 *}$, ZUFENG HUANG $^{1,2}$, \\ YI QI ${ }^{4}, \mathrm{YUAN} \mathrm{HE}^{1}, \mathrm{CAN} \mathrm{CHEN}^{1}$, HUI LUO ${ }^{4}$ and WEI LEI ${ }^{1,2,4}$ \\ ${ }^{1}$ Laboratory of Cardiovascular Diseases, Guangdong Medical University; ${ }^{2}$ Cardiovascular Medicine Center, \\ Affiliated Hospital of Guangdong Medical University; ${ }^{3}$ Laboratory of Vascular Surgery, Guangdong Medical University; \\ ${ }^{4}$ Southern Marine Science and Engineering Guangdong Laboratory (Zhanjiang), The Marine Biomedical Research Institute, \\ Guangdong Medical University, Zhanjiang, Guangdong 524001, P.R. China
}

Received February 20, 2020; Accepted May 25, 2020

DOI: $10.3892 /$ ijmm.2020.4674

\begin{abstract}
Cardiovascular diseases (CVDs), such as atherosclerosis, hypertension, myocardial infarction and diabetic heart disease, are associated with high morbidity and mortality rates worldwide, and may also induce multiple organ failure in their later stages, greatly reducing the long-term survival of the patients. There are several causes of CVDs, but after nearly a decade of investigation, researchers have found that CVDs are usually accompanied by an imbalance of gut microbiota and a decreased abundance of flora. More importantly, the metabolites produced by intestinal flora, such as trimethylamine and trimethylamine $\mathrm{N}$-oxide, bile acids, short-chain fatty acids and aromatic amino acids, exert different effects on the occurrence and development of CVDs, as observed in the relevant pathways in the cells, which may either promote or protect against CVD occurrence. It is known that changes in the intestinal flora following antibiotic administration, diet supplementation with probiotics, or exercise, can interfere with the composition of the intestinal flora and may represent an effective approach to preventing or treating CVDs. The focus of this review was the analysis of gut microbiota metabolites to elucidate their effects on CVDs and to identify the most cost-effective and beneficial methods for treating CVDs with minimal side effects.
\end{abstract}

Correspondence to: Dr Wei Lei, Laboratory of Cardiovascular Diseases, Guangdong Medical University, 57 Renmin Southern Road, Xiashan, Zhanjiang, Guangdong 524001, P.R. China E-mail: leiwei2006@126.com

Dr Hui Luo, Southern Marine Science and Engineering Guangdong Laboratory (Zhanjiang), The Marine Biomedical Research Institute, Guangdong Medical University, 57 Renmin Southern Road, Xiashan, Zhanjiang, Guangdong 524001, P.R. China

E-mail: luohui@gdmu.edu.cn

${ }^{*}$ Contributed equally

Key words: cardiovascular diseases, gut microbiota, metabolites, pathways, treatment

\section{Contents}

1. Introduction

2. Association between gut microbiota metabolites and angiocardiopathy

3. Targeted regulation of CVDs by several prime metabolites from gut microbiota

4. Treatment of CVDs based on intestinal microflora and their metabolites

5. Conclusion

\section{Introduction}

According to global statistical reports, 17.5 million individuals succumb to cardiovascular diseases (CVDs) annually worldwide (1). In recent years it has been found that dysfunctional microflora-dependent intestinal metabolism is a risk factor for CVDs and is relevant to the development of various angiocardiopathies, including stroke, hypertension, atherosclerosis and myocardial dysfunction (2). With modern-day changes in living conditions, CVDs are no longer considered geriatric diseases, as they also affect younger age groups (3).

The pathogenic mechanisms underlying the development of CVDs have both genetic and environmental elements. CVD is not a single-factor disease, as it is often affected by multiple factors, such as hyperglycemia (4), hyperlipidemia (5) and hormonal dysfunction (6), further complicating the understanding of this disease. The clinical methods for diagnosing and treating CVDs are continuously improved and updated, and the association between intestinal flora and CVD has become a research hotspot in recent years, with researchers finding that anaerobic bacteria constitute the main intestinal flora, with 500-1,000 recognized species to date (7). It has been found that imbalances in intestinal flora are associated with various CVDs in humans, and there is evidence that metabolites regulate the development of CVDs through inflammation, apoptosis and other signaling pathways $(8,9)$.

Intestinal flora produces a number of metabolites, but trimethylamine (TMA) and trimethylamine $\mathrm{N}$-oxide (TMAO) (10), bile acids (BAs) (11), short-chain fatty acids 
(SCFAs) (12) and aromatic amino acids (AAAs) (13) are considered the most important for cardiac and vascular function in humans (Fig. 1).

\section{Association between gut microbiota metabolites and angiocardiopathy}

Angiocardiopathies, which include coronary atherosclerotic cardiopathy, diabetic cardiomyopathy, heart failure, stroke, hypertension and peripheral vascular disease, are a group of diseases affecting the heart and blood vessels (14). Over the last few decades, dietary and lifestyle modifications have been focused on lowering or eliminating modifiable angiocardiopathy risk factors, such as hypercholesterolemia, hyperlipidemia, hypertension, type 2 diabetes mellitus, or smoking (15). Consequently, although overall mortality from CVDs has declined over this time period, angiocardiopathy remains the main cause of death across the globe (16).

The digestive tract is colonized to different degrees by intestinal microbes that play a key role in digestive physiology and intestinal homeostasis in humans and other mammals, and their role in metabolism is associated with carbohydrate and protein digestion (17). The gut microbiome functions like an endocrine organ, with colonic microorganisms mediating the final stages of the digestive process, which may impact host physiology (18). The intestinal tract continuously absorbs the low-molecular-weight metabolites produced by intestinal flora, which are transported to the liver for processing and release into the systemic circulation. These metabolites act on several sites in the body and may be beneficial and/or harmful to the host by, for example, promoting or reducing the risk of CVDs (19). Intestinal homeostasis imbalance, immune dysfunction, environmental and other factors may cause a number of diseases, and intestinal microflora plays an important role in maintaining a healthy intestinal balance. Recent studies indicated that gut dysbacteriosis may be associated with the occurrence and development of atherosclerosis, myocardial infarction, hypertension, diabetes and hyperlipidemia, and that microbiota metabolites may play a protective or aggravating role in angiocardiopathies $(18,20)$. The majority of studies in the field of metabolic diseases has been focused on the role of chronic inflammation (21). Wang et al (22) found that intestinal flora participates in the etiopathogenesis of cardiovascular and metabolic diseases, suggesting that the microbiome may represent a target for the treatment of metabolic diseases. However, the mechanisms and impact of intestinal flora in the pathogenesis of disease and its complications have yet to be fully elucidated.

\section{Targeted regulation of CVDs by several prime metabolites from gut microbiota}

The gut flora may be subdivided into three broad categories: Beneficial, harmful and neutral bacteria (23). The gut microbiota participates in host metabolism by interacting with host signaling pathways, such as the TMA or TMAO, SCFA, primary and secondary BA and phosphatidylcholine pathways $(10,24)$. The metabolites mentioned above may be pro-inflammatory, or protective and anti-inflammatory, or play a largely unknown biological role. Therefore, it is important to identify new factors implicated in the occurrence and development of diseases associated with the effect of gut microbiota on such pathways. Hence, the focus of the present review was our current knowledge on the three most extensively investigated metabolites produced by intestinal flora.

Associations between choline, TMA, TMAO and cardiovascular risk factors. Choline is the precursor of phosphatidylcholine, sphingomyelin, acetylcholine and betaine, and also participates in signaling and lipid transport, one-carbon metabolism, neurotransmission and membrane structure (25). Of note, choline, phosphatidylcholine and carnitine are metabolized by intestinal flora to produce TMA, which is transformed in the liver into TMAO by flavin monooxygenase 3 (FMO3) (26). TMAO, a small quaternary amine that directly induces conformational changes in proteins, stabilizes protein folding and acts as a small molecular protein chaperone (27), whereas TMA can affect signal transduction by directly interacting with a family of GPRs. More importantly, it can upregulate the scavenger receptors CD36 and SRA on the surface of macrophages, thereby promoting the accumulation of cytoplasmic cholesterol and accelerating the development of atherosclerosis $(28,29)$. TMAO may accelerate platelet activation and inflammation, the levels of which are increased in atherosclerosis and associated complex CVDs (30).

Brown and Hazen discovered that atherosclerosis-prone mice fed choline- and TMAO-rich diets exhibited accelerated development of atherosclerosis and cholesterol metabolism disorders (31). The main mechanism by which TMAO promotes atherosclerosis is the reverse transport of cholesterol and its catabolism, and atherosclerotic plaques containing large amounts of bacterial DNA have been confirmed by autopsy $(32,33)$. This evidence suggests that the chronic inflammation caused by intestinal flora promotes the formation of atherosclerotic plaques. Clinical cohort studies have demonstrated that in vivo choline, TMAO and betaine levels may be used as predictors of short- and long-term malignant cardiovascular events (31). However, Winther et al (34) analyzed 1,159 patients with type 1 diabetes and found that high plasma TMAO concentrations increased the risk of cardiovascular events, renal vascular disease and mortality in these patients, indicating that TMAO damages the microand macrovasculature, leading to renal failure (22). In recent years, researchers have come to realize that several of the pathways involved in inflammation, apoptosis, pyroptosis and autophagy are involved in regulating intestinal flora and its metabolites, which may have a positive or negative impact on the body. In mouse experiments, $\mathrm{Li}$ et al observed that TMAO can activate the toll-like receptor 4 (TLR4)-NLR family pyrin domain containing 3 (NLRP3)-transforming growth factor- $\beta$ (TGF- $\beta$ ) signaling pathway in vivo as well as in vitro, leading to an increase in the production of reactive oxygen species, inflammatory cytokines and collagen deposition, and resulting in severe cardiac fibrosis and cardiac dysfunction (35). TMAO also induces proliferation, migration and collagen synthesis in cardiac fibroblasts. Possible mechanisms underlying the role of TMAO in angiocardiopathy were proposed recently: TMAO may be involved in homeostatic balance in the endoplasmic reticulum, as shown by an experiment where overexpression of the green fluorescent protein-tagged iodide transporter 


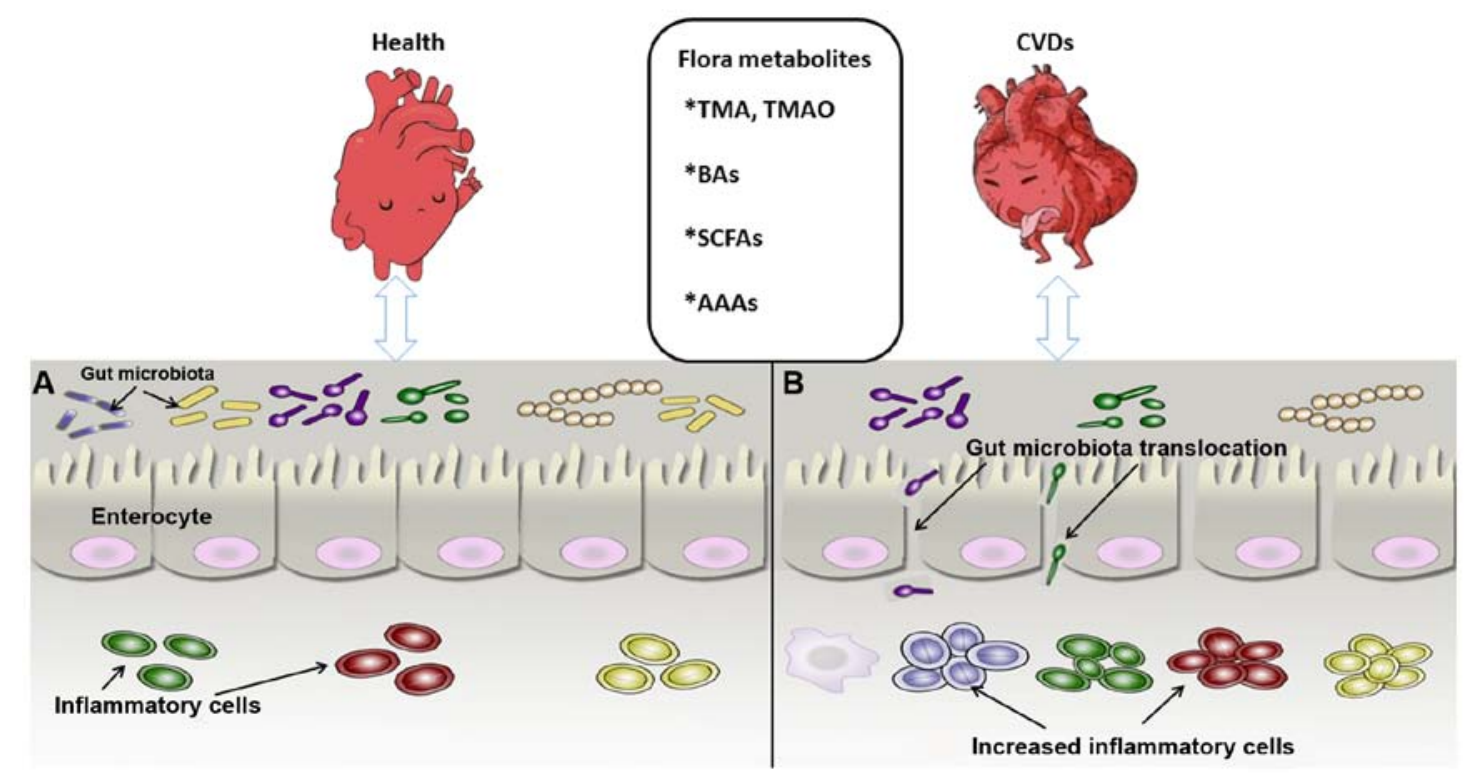

Figure 1. Association between intestinal flora metabolites and CVDs. (A) Gut microbiota and cardiovascular system in the healthy state. The microbial biomass increases, with the inflammatory cells maintaining a normal defense function and the intestinal epithelial cells remining tightly connected to maintain the mucosal barrier. (B) Intestinal flora state under CVD condition. The microbial biomass is reduced significantly and the intestinal epithelium permeability increases, leading to translocation of intestinal bacteria and marked proliferation of inflammatory cells, which impairs intestinal mucosal barrier function and may causes CVDs and metabolic syndrome, among others. CVDs, cardiovascular diseases; BAs, bile acids; SCFAs, short-chain fatty acids; AAAs, aromatic amino acids; TMA, trimethylamine; TMAO, trimethylamine N-oxide.

pendrin caused endoplasmic reticulum perturbation (36). The endoplasmic reticulum stress kinase (PERK) is recognized by TMAO and stimulates the PERK embranchment of the unfolded protein response, causing dysfunction of glucose metabolism; hence, targeting the TMAO-PERK pathway may improve metabolic disorders (37). Moreover, TMAO has been shown to accelerate endothelial cell dysfunction by activating the protein kinase $\mathrm{C}(\mathrm{PKC}) /$ nuclear factor $-\kappa \mathrm{B}(\mathrm{NF}-\kappa \mathrm{B}) /$ vascular cell adhesion molecule 1 (VCAM-1) pathway during the pathological process of atherosclerosis (38). Therefore, altering the abundance of intestinal flora and inhibiting the production of TMAO may be of value in preventing and treating CVDs.

Interaction of gut microflora with BA metabolism and its impact on disease states. BAs are synthesized in the liver from cholesterol via the classic and alternative pathways (39) (Fig. 2), and they are recycled mainly through the enterohepatic circulatory system (40). The classic pathway undergoes a series of reactions to produce diols and triols, which are carboxylated by mitochondrial CYP27A1 to produce cholic acid $(3 \alpha, 7 \alpha, 12 \alpha$-trihydroxy-5 $\beta$-cholic acid; CA) and chenodeoxycholic acid ( $3 \alpha, 7 \alpha$-dihydroxy-5 $\beta$-cholic acid; CDCA), the latter of which is the primary BA formed in the human body. These two primary BAs combine with taurine or glycine to form secondary BAs, which are subsequently excreted into the bile. Alternating hydroxylation reactions produce primary BAs with different structures, particularly $\alpha-, \beta$ - and $\gamma$-polyphenolic acids. Therefore, under the action of intestinal flora, the chemical diversity of the BA pool in the body increases, and CA and CDCA produce two major secondary BAs, deoxycholic acid (3 $\alpha, 12 \alpha$-dihydroxy-5 $\beta$-cholic acid; DCA) and lithocholic acid ( $3 \alpha$-hydroxy- $5 \beta$-cholic acid; LCA), respectively. CYP7A1, a microsomal cytochrome P450 isozyme, catalyzes the first and rate-limiting steps of this pathway. Nuclear receptors and genes regulated by BAs/oxysterol are potential targets for the treatment of cardiovascular and liver diseases and the reduction of triglyceride and cholesterol levels $(40,41)$. The bile stored in the gallbladder is stimulated for release into the intestine postprandially (42). Intestinal flora modulation of BAs is pivotal to producing DCA and LCA (43). Most BAs are reabsorbed at the end of the ileum and upper colon by the apical sodium-dependent BA transporter and the ileal BA transporter in the intestinal cells (44). BAs play vital roles in lipid and glucose metabolism and energy consumption, and have also emerged as newly identified metabolic regulators of signaling molecules (45).

Gut bacteria affect BA synthesis by regulating the expression of the farnesoid $\mathrm{X}$ receptor (FXR) and G protein-coupled receptors (GPRs) (46). Numerous experiments have confirmed that FXR is a BA receptor that is expressed in the aorta and cardiomyocytes. BAs may compromise cell membrane and DNA integrity, and/or induce protein degeneration and inactivation (47). BAs inhibit the growth of intestinal flora through the FXR target system (48), and FXR plays a major role in protecting the distal small intestine from bacterial invasion. Activation of FXR may prevent translocation of intestinal flora. It was previously reported that the total BA (TBA) levels in the serum of patients with hypertension are directly proportional to the severity of the disease (41). BAs may be involved in the occurrence and progression of hypertension through $11 \beta$-hydroxysteroid dehydrogenase (11 $\beta$-HSD) (49). This enzyme can rapidly inactivate cortisol and prevent excessive cortisol and aldosterone from competing for mineralocorticoid receptors. However, when the expression of $11 \beta$-HSD is deficient or suppressed, blood pressure (BP) may increase (50). For patients with coronary atherosclerotic disease caused by a high cholesterol diet (51), BA excretion disorders may significantly reduce the excretion of TBAs, DCA and LCA (52). GPR5 is an 


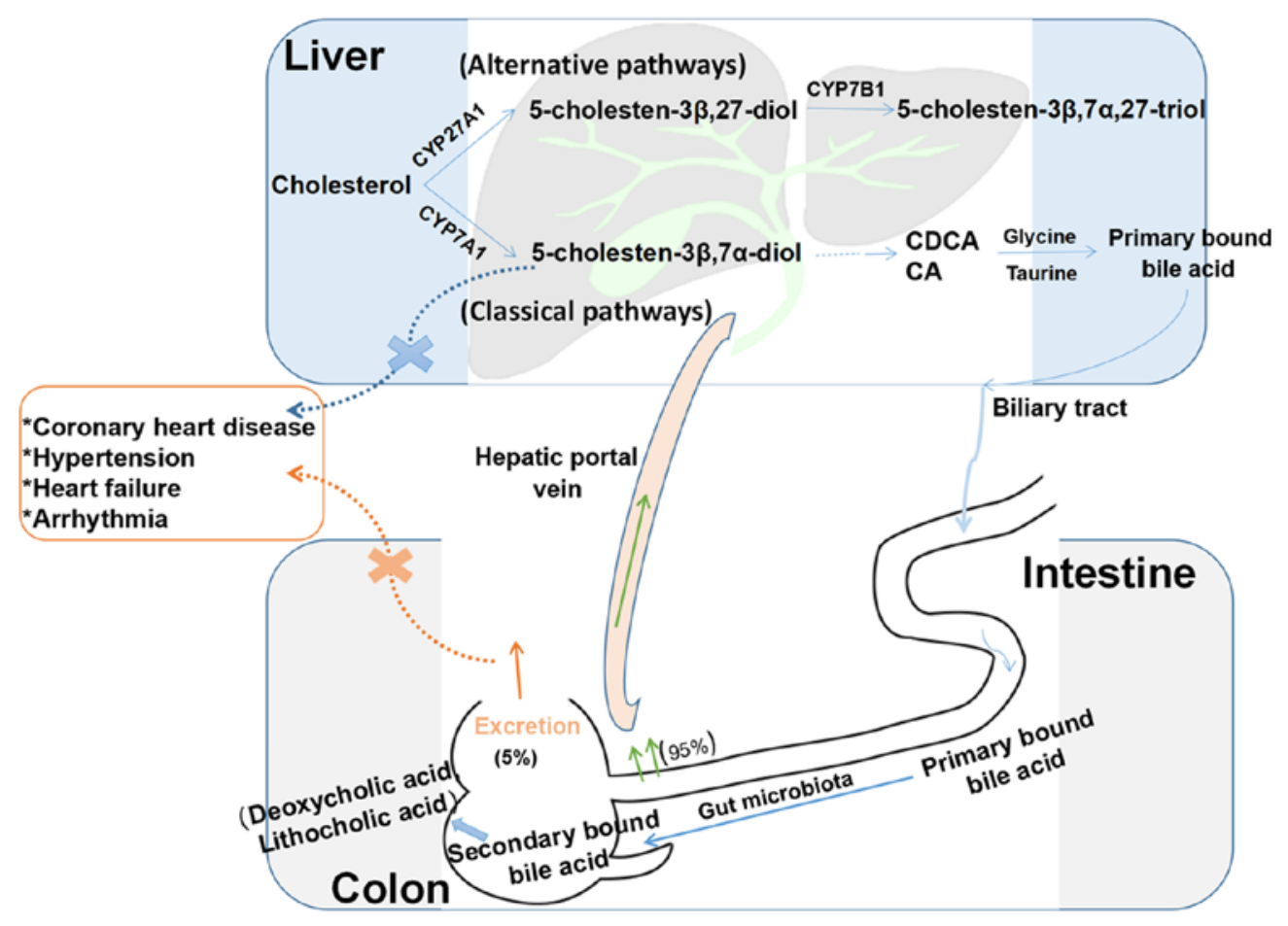

Figure 2. Enterohepatic circulation of BAs. BAs are synthesized from cholesterol in the liver through classic and alternative pathways. The classic pathway includes a series of reactions to produce diols and triols, which are carboxylated by mitochondrial CYP27A1 to produce the primary BAs CA and CDCA. These two BAs combine with taurine or glycine to form primary conjugated BAs, which are then secreted from the biliary tract into the intestinal cavity and metabolized by the intestinal flora to form secondary BAs (deoxycholic acid and lithocholic acid). Approximately 95\% of BAs are reabsorbed in the terminal ileum and ascending colon, and approximately $5 \%$ are excreted in the feces. The alternative pathway includes a series of enzymatic reactions to form 5 -cholesten-3 $\beta, 7 \alpha, 27$-triol. When 5-cholesten-3 $\beta, 7 \alpha$-diol production disorders or secondary BA excretion disorders occur, they may lead to coronary heart disease, hypertension and other cardiovascular diseases. BAs, bile acids; CA, cholic acid; CDCA, chenodeoxycholic acid.

intracellular receptor for BAs, and intestinal flora can inhibit the synthesis of BAs by activating GPR5 expression. Therefore, BAs and their derivatives activate the anti-atherosclerotic effect of FXR. Thus, the BA content in plasma may be used as a predictive factor for CVD risk (24). In vitro electrophysiological experiments have confirmed that different BAs exert various effects on cardiomyocytes, mainly by altering the concentrations of sodium, potassium and calcium. Hydrophilic BAs (e.g., ursodeoxycholic acid) may play a role in stabilizing the cell membrane potential and preventing arrhythmia (53). Lipophilic BAs readily induce changes in membrane potential, exert toxic electrophysiological effects on cell membranes, and facilitate the occurrence of arrhythmias (54). It was also found that cardiomyocyte apoptosis occurs after free BAs activate FXR and caspase-9/caspase-3, regulate BCL-2/BAX expression, cause mitochondrial dysfunction, and ultimately lead to ischemia-reperfusion injury in myocardial cells (55). Certain antibodies can reduce TMAO synthesis by blocking the choline-TMA pathway, thereby reducing the incidence of atherosclerosis (56). The interaction between BAs and gut intestinal flora exerts important regulatory effects on CVDs, and may prove useful in guiding treatment.

Crosstalk between gut microbiota and SCFAs, and modulation of the mechanisms involved in CVDs. Fatty acids (FAs), which are carboxyl-containing, long-chain aliphatic hydrocarbons, are a staple component of glycolipids, phospholipids and neutral fats. Obesity caused by a high-fat diet (HFD) increases the risk of CVD; however, the type of FA consumed has a more pronounced effect on the disease (56). In fact, the levels of eight metabolites in the intestinal flora are known to undergo significant changes with such diets; these include ethylmethylacetic acid, butyric acid, valeric acid, palmitic acid, stearic acid, arachidonic acid, indole and indoleacetic acid, the latter of which increases sputum production. Stearic and palmitic acids, which are the main saturated FAs, are believed to stimulate inflammatory signaling by macrophages and readily cause chronic vascular inflammation in vivo and in vitro $(51,57)$.

According to the data released in 2015 relating to medical office BP measurements, hypertension has a global prevalence of 1.13 billion and 150 million cases in Central and Eastern Europe, respectively. In recent years, studies (58) have found that dysbiosis of the intestinal flora affects BP stability. Metabolites from microbial flora, such as SCFAs and TMAO, are implicated in the pathogenesis of CVD. Therefore, it has been suggested that the metabolic products from microflora may be used as targets to treat hypertension (58). SCFAs, which are mainly produced by anaerobic bacteria, such as Lactobacillus and Bifidobacteria, are an important energy source for intestinal microflora and the intestinal epithelial cells of the host, and can inhibit the growth of harmful bacteria, maintain the acid-base balance in the intestinal tract, reduce inflammatory responses, and regulate the host's intestinal immunity. Butyric acid, acetic acid and propionic acid constitute $90 \%$ of the intestinal SCFAs (59). They are absorbed into the blood circulation, providing energy for the heart, brain, muscles and kidneys, and affect the transport and metabolism of epithelial cells (60). Two studies have reported that some 
Anaerostipes and other eubacterial species utilize D- and L-lactate to generate acetate, propionate and butyrate $(15,17)$. Experiments have revealed that SCFAs can regulate the immune response by activating GPRs and inhibiting histone deacetylases (HDACs), as well as partially suppressing inflammation. GPR is expressed in almost all immune cells, and SCFAs mainly activate GPR41, GPR43 and GPR109 (61). It was recently discovered that, in type 2 diabetes, the intestinal metabolite sodium butyrate $(\mathrm{NaB})$ maintains blood glucose homeostasis and expedites glycogen metabolism by adjusting the GPR43-protein kinase (Akt)-glycogen synthase kinase (GSK) 3 signaling pathway, and the results from experiments in $\mathrm{db} / \mathrm{db}$ mice have shown that $\mathrm{NaB}$ administration can reduce glycogen staining in cells and improve hepatic lobular fibrosis and steatosis, thereby decreasing the damage to systemic blood vessels caused by hyperglycemia (15). HDACs are a group of proteins involved in modifying chromosome structure and regulating gene expression. The degree of deacetylation of Foxp3 is affected by HDAC9, which is prone to Foxp3 degradation. SCFAs may also be used by the body through HDAC inhibition to enhance histone acetylation of Foxp3, promote the differentiation of $\mathrm{T}$ cells into effector and regulatory $\mathrm{T}$ cells, and inhibit the inflammatory response (62). Moreover, SCFAs have been shown to affect olfactory receptors in blood vessels and kidneys, thereby affecting BP (63). A cross-sectional study on BP and intestinal flora in hypertensive subjects revealed that some SCFA-producing microbes (Lachnospiraceae, Bacteroides plebeius and Bacteroides coprocola) were more highly abundant, while others (Roseburia hominis, Faecalibacterium prausnitzii, Ruminococcaceae NK4A214, Christensenellaceae_R-7 and Ruminococcaceae_UCG-010) were less abundant (58). These species are positively correlated with systolic and diastolic BP. The same study reported that the level of SCFAs in the plasma was low, whereas the level of fecal SCFAs was significantly higher, indicating that the intestinal SCFA absorption rate is low (58). The SCFA content is positively correlated with Clostridium, Lactobacillus and Blautia XIVa levels, whereas acetic acid, propionic acid and butyric acid levels are inversely proportional to the abundance of Streptococci, Enterococci and other bacteria (22).

Thus, it appears that SCFAs can enhance the abundance of intestinal flora and improve the numbers of beneficial flora, so as to maintain the metabolic balance of sugars, lipids and proteins and reduce the occurrence of CVDs, diabetes and hypertension.

Gut microbiota-derived AAAs and types of angiocardiopathy. AAAs, such as tyrosine (Tyr), tryptophan (Trp) and phenylalanine (Phe), are benzene ring-containing amino acids. Among them, Trp and Phe must be obtained from the diet, whereas Tyr may be obtained by Phe hydroxylation in the human kidney and liver (64). Dietary proteins, such as fish, beef, pork and chicken, are the main sources of AAAs (65). Tyr and Trp are the precursors of a large number of bioactive molecules: Dopamine, epinephrine, norepinephrine and thyroid hormone are derived from the former, and serotonin, melatonin and niacin from the latter $(66,67)$.

Dodd et al (45) characterized a pathway whereby the metabolites of AAAs were generated by the gut symbiont Clostridium sporogenes, and all three AAAs were used as substrates in this pathway. The authors demonstrated that Clostridium sporogenes, a type of intestinal bacteria from the Firmicutes phylum, can regulate AAA metabolism through the fldC gene and metabolize dietary Trp into indolepropionic acid (IPA), which accumulates in the serum and exhibits wide differences between individuals. IPA affects intestinal epithelial permeability and systemic immunity by acting directly on the progesterone $\mathrm{X}$ receptor (45).

Shishehbor et al determined the levels of 3-nitrotyrosine in the plasma from patients with coronary heart disease via stable isotope-dilution high-performance liquid chromatography and on-line electrospray ionization tandem mass spectrometry (67). They found that modified apolipoprotein A-1, apolipoprotein B-100, fibrinogen and nitrated Tyr residues promote atherosclerosis, which may be a risk factor for thrombosis (68). Metabolites from AAAs (such as Trp) inhibit inflammation by upregulating the aryl hydrocarbon receptor (AhR) in astrocytes or T-cells; however, excessive intake of foods containing long-chain FAs is likely to affect the production of metabolites (69). AhR is a hydrocarbon with a benzene ring structure and, as a ligand-induced transcription factor, it is expressed in both immune and epithelial cells (70). It is known that Trp is an essential amino acid that it is metabolized by intestinal flora to produce immunoregulatory products that can bind to AhR. Among these, Trp-positive bacterial species of Lactobacillus can produce various indole metabolites from dietary Trp, activating AhR and inhibiting inflammation (71). Indoleamine 2,3-dioxygenase is an enzyme induced in numerous types of immune cells (e.g., macrophagocytes) in response to inflammatory stimuli, thereby facilitating the degradation of Trp along the kynurenine pathway; however, when the activity of this enzyme is high, it may worsen angiocardiopathy and accelerate vascular inflammation and atherosclerosis (72). Additionally, obesity is known to be associated with an increase in enteric canal indoleamine 2,3-dioxygenase activity (73). When this enzyme is inhibited or the gene encoding it is knocked out, insulin sensitivity improves, the intestinal mucosal barrier is preserved, and endotoxemia and chronic inflammation improve, while lipid metabolism in adipose tissues and the liver is regulated (73). Therefore, the products of AAA catabolism extracted from the intestinal flora may be used as biomarkers for imbalances in intestinal flora, an may also serve as targets for intestinal flora development (74). Hoyles et al performed rodent and human hepatocyte experiments to combine metagenomic sequences with metabolomics data to identify the mechanism(s) through which AAAs exert their biological effects. A direct role for gut-derived degradation of AAAs from microorganisms in peripheral arterial disease into phenylacetic acid has been shown to involve increased branched-chain amino acid (BCAA) utilization and AAA metabolism to promote steatosis. Circulating fat metabolism indicators support BCAA metabolism and microbial metabolism of AAAs such as Phe, Tyr and Trp in liver steatosis (75).

\section{Treatment of CVDs based on intestinal microflora and their metabolites}

Intestinal microbes are used to study the pathogenesis and determine the direction of treatment for CVDs. Research in 


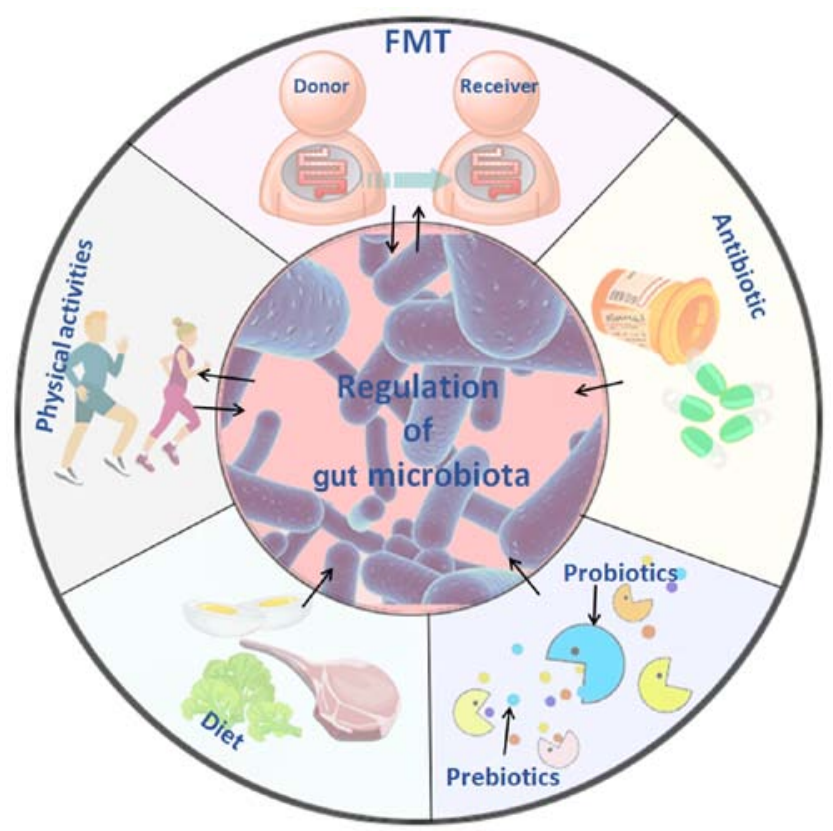

Figure 3. Treatment of cardiovascular diseases based on intestinal microflora and its metabolites. Five treatment methods that are closely associated with intestinal flora are depicted: FMT involves transplanting the intestinal flora of healthy individuals into the patient, and it may delay or prevent developing the disease through the effects of the flora or its metabolites. The use of antibiotics may alter the structure and abundance of intestinal flora, inhibit the proliferation of harmful flora, and improve the progression and prognosis of cardiovascular diseases to a certain extent. Probiotics, such as Bifidobacterium species and lactic acid bacteria species temporarily colonize the intestine, and change the intestinal environment by affecting cardiovascular disease-related pathways and metabolites; prebiotics can selectively stimulate the growth of predominant bacteria in the intestine, improve glucose and lipid metabolism and cooperate with probiotics improve the gut microbiome. Changes in diet composition and habits lead to changes in the metabolic function of intestinal flora, and the reduction of harmful metabolites, such as TMAO, may reduce the incidence of cardiovascular disease. Physical activities of different types and intensities affect the abundance and metabolites of intestinal flora, increase the abundance of beneficial flora, and reduce the high-risk factors that cause cardiovascular disease. TMAO, trimethylamine N-oxide; FMT, fecal microbiota transplantation.

this area has focused on fecal transplantation, the antibacterial effect of antibiotics, probiotics and prebiotics, but systematic research in this area remains relatively scarce. The treatment options and methods focusing on physical activity and dietary regulation are shown in Fig. 3.

Approaches and rationale for the use of fecal microbiota transplantation (FMT). FMT has been a hot research topic in recent years, and new discoveries have been made in disease treatment and the mechanisms regarding its use. FMT refers to the transfer of microbial communities from healthy donors to patients and has become an effective treatment option for chronic diseases (76). Studies have shown that the composition of the flora in the intestine may be associated with CVDs such as atherosclerosis, hypertension and heart failure. An imbalance in intestinal flora may cause metabolic disorders and the release of endotoxins, which may ultimately exacerbate the occurrence and development of CVDs (77).

In a cohort study using FMT from patients to germ-free mice (78), it was observed that, compared with healthy controls, microbial richness and diversity were markedly decreased, and that the Prevotella genus dominated the gut enterotype, whereas reduced numbers of Prevotella bacteria were associated with a healthy status, and overgrowth of bacteria such as Prevotella and Klebsiella was observed in metagenomic and metabolomic analyses (79). A number of microbial CVD-specific biomarkers have been identified, and fecal microbiome-targeted strategies are currently considered as a powerful tool for early diagnosis and treatment of different diseases. More importantly, through fecal transfer experimentation and gut microbiota remodeling, the intestinal microbiome has been confirmed to be involved in the pathogenesis of multiple diseases, such as obesity, depressive disorders, chronic ileal inflammation, liver diseases and atherosclerosis (63). A previous study indicated that there are clinical and mechanistic links between atherosclerotic heart disease and the microbial metabolism of certain dietary nutrients producing intestinal TMAO, and verified that intestinal microbial transplantation may enhance choline-induced TMAO production and susceptibility to atherosclerosis (78). The findings mentioned above indicate that, when intestinal flora from a subject with CVD is transplanted into a healthy organism, it may result in the development of angiocardiopathy, thus confirming that alterations in intestinal microflora play a pivotal role in driving the development of CVDs $(21,63)$. FMT from patients with type 2 diabetes to germ-free mice revealed that the microbiome-induced modulation of the dipeptidyl peptidase-4 inhibitor (DPP-4i) contributed to its hypoglycemic effect, and that the DPP-4i-altered microbiome improved glucose tolerance in the colonized mice, while acarbose did not (80). Moreover, DPP-4i increased the abundance of Bacteroidetes, and also promoted a functional shift in the gut microbiome, particularly by increasing the production of succinate (80). This suggests that transplanting the gut flora from a diseased into a healthy organism may promote disease development and that, in turn, transplanting the gut flora from a healthy into a non-healthy organism may alleviate or even cure the disease. 
Antibiotics influence the effect of intestinal flora and related metabolites on CVD. Antibiotics have been used clinically to treat microbial infections, and their long-term use, misuse, or abuse are associated with a variety of side effects in the recipients. Wan et al (81) reported that the early administration of antibiotics or FMT may reduce the oxidative catabolism of FAs and amino acids in newborn piglets. The synthesis of such acids may represent a point of reference for regulating host metabolism.

Lam et al detected intestinal flora metabolites by mass spectrometry after feeding rats with broad-spectrum antibiotics, and found that the catabolism of AAAs was affected the most. Furthermore, antibiotic use has been shown to reduce the incidence of myocardial infarction in vivo, indicating that the intestinal flora affects the severity of myocardial infarction in rats. In addition, vancomycin protects the heart by activating Janus kinase-2, Akt/phosphoinositide 3-kinase, p44/42 mitogen-activated protein kinase (MAPK) and p38 MAPK pathways and $\mathrm{K}_{\text {ATP }}$ channels (19). Antibiotics can inhibit the metabolism of L-carnitine into TMA in intestinal flora, thereby reducing the synthesis of TMAO, reducing its stimulation of macrophages and the vascular endothelium, and decreasing the incidence of atherosclerosis (82).

A direct association between intestinal flora and the severity of myocardial infarction induced in rats has been highlighted by statistical analysis (83). In addition, Le Roy et al (84) used spectral antibiotics to inhibit the intestinal flora in $\mathrm{ApoE}^{-/}$mice with hypercholesterolemia. The authors found that intestinal flora in the mice exhibited a significant correlation with cholesterol regulation. Furthermore, samples of intestinal flora from patients with hypercholesterolemia were transplanted into experimental mice, which induced a plasma cholesterol phenotype in these mice. Some researchers have evaluated the role of intestinal flora transplantation as a complementary therapy for obesity, and found that it may reverse the effects of antibiotics and re-establish balance in the microbiome, thus restoring normal function and improving the diversity of the microbiome (85). Experiments have shown that controlling plasma cholesterol levels is key to preventing CVDs, and that gut flora can determine the levels of circulating cholesterol. This may provide a new method for supporting health and dietary habits as a first choice for maintaining homeostasis in the microbiome (86). In another study, antibiotic therapy has been shown to alleviate glucose intolerance, inflammation and liver steatosis caused by HFD, along with reduced liver lipogenesis and increased heat production in subcutaneous white adipose tissue (43). The diversity of intestinal flora in HFD mice and mice treated with antibiotics in their drinking water was significantly reduced, altering the function of the flora in the host (79).

Of note, antibiotic treatment may change the absorption rate, BA pool and bioavailability of drugs (87). The use of antibiotics to treat CVDs remains controversial due to their long-term side effects, and their use may decrease intestinal flora abundance and promote antibiotic resistance.

The cardiovascular system, probiotics, prebiotics and intestinal flora. Probiotics are a class of active microorganisms that are beneficial to the host. After colonizing the human body, they alter the composition of the flora in the host to a certain degree (88) by promoting nutrient absorption and maintaining intestinal health by regulating host mucosal and systemic immune function or by regulating the balance of the intestinal flora (89). Probiotics include bacteria and yeast that act in the small and large intestine. Most probiotics include certain strains of Escherichia coli, Bifidobacteria species, Lactobacillus species, Lactococcus lactis, Streptococcus species and some Enterococcus species, with Saccharomyces boulardii being the most commonly used type of yeast. Based on the fecal persistence of the ingested strain, probiotics appear to temporarily settle in the gut and act by altering the colonic environment. Probiotics work in different ways through direct or indirect regulation in the host. First, they enhance the barrier function of the gastrointestinal tract through the tight junction proteins of the intestinal epithelium and the mucins secreted by goblet cells. Second, some probiotics can produce 'bacteriocin', SCFAs and other antimicrobial factors that inhibit pathogen growth. Third, probiotics regulate the phenotype and activity of $\mathrm{T}$ cells, natural killer cells and macrophages, and reduce proinflammatory factor release by regulating the NF-kB pathway (90).

Prebiotics are non-digestible but fermentable dietary supplements that can improve the host's health by selectively stimulating the growth and activity of one or more dominant colonies already present in the colon, but are not limited to Lactobacillus and Bifidobacterium (91). A separate meta-analysis of a randomized clinical trial demonstrated that the intake of probiotics and prebiotics in foods or supplements significantly improved blood glucose levels, insulin levels and insulin resistance (92). Resveratrol (RSV) has been found to significantly regulate the growth of certain intestinal flora, including increasing the ratio of Bacteroidetes to Firmicutes, as well as the growth of Bacteroides, Lactobacillus and Bifidobacterium. This indicates that RSV may be a good candidate as a prebiotic that may be used to promote the growth of flora that can confer health benefits to the host (56). Galacto-oligosaccharides (GOS), which are common prebiotics, may participate in the regulation of lipid metabolism and are beneficial to the intestinal flora. In experimental studies on Sprague-Dawley rats, administration of GOS and fucoidan (a complex polysaccharide) improved dyslipidemia and exerted a positive effect on total cholesterol and triglyceride levels in overweight adults, and reduced hepatic steatosis and aortic arch atheroma formation (93). GOS may also reduce total serum cholesterol by regulating the microbiome in infants (93). Guar gum, which is also a type of prebiotic, may change the composition of intestinal flora in rodent models of HFD, thereby reducing diet-induced obesity and improving glucose tolerance, but it may worsen the liver phenotype, leading to increased inflammation and fibrosis in a HFD model (23). Probiotics or prebiotics may be used as therapeutic agents for BA-related metabolic disorders, suggesting that microbiome-based therapies may be effective in preventing and/or treating intestinal-related diseases, including atherosclerosis (94).

Probiotics and prebiotics also improve the accumulation of toxins produced by harmful intestinal bacteria in the body by regulating the abundance of intestinal bacteria and the interactions between symbiotic bacteria. In the clinical setting, there are few reliable statistical analyses on the safety and side 
effects associated with the use of probiotics and prebiotics; therefore, the medicinal value of microbial therapy requires further investigation.

Physical activity may positively modulate gut microbiota in CVDs. Proper physical activity is known to boost metabolism and immunity. However, with the increasing scope of research in the field of intestinal flora, it is suspected that exercise affects the regulation of intestinal flora and the extent to which intestinal flora impacts disease prevention and progression. Maintaining homeostatic balance in the intestinal flora is now considered to support the health of the host. It has been demonstrated that physical activity can independently reduce the risk of coronary heart disease and other CVDs (94), such as hypertension, hyperlipidemia, obesity and diabetes $(95,96)$. Vascular wall shear stress during exercise improves endothelial cell function and can lower serum C-reactive protein levels (97).

Aerobic capacity, which is expressed as peak oxygen consumption ( $\mathrm{V}_{\mathrm{O} 2}$ peak), is the gold standard used for predicting cardiovascular health and all-cause mortality, even for patients who already have various CVDs and coronary risk factors (98). Cardiopulmonary fitness is associated with increased intestinal flora diversity in healthy individuals, and the intestinal flora of hypertensive patients is significantly imbalanced with a reduced abundance of certain flora. Among these microbes, opportunistic genera, such as Streptococcus and Klebsiella, are common, whereas others, such as Clostridium tenella, are more common in healthy individuals. Although the intrinsic mechanism through which intestinal flora regulate $\mathrm{BP}$ remains unclear, SCFAs play a key role in the pathogenesis of hypertension. SCFAs produced by the intestinal flora regulate BP through Olfr78 (an olfactory receptor) and Gpr41 (99). High-throughput 16S rDNA sequencing analysis of intestinal flora uncovered that, when healthy individuals exercised, their Bacteroides and Bifidobacteria levels and SCFAs increased significantly (83), helping to maintain the dynamic balance of the intestinal immune system and, to a certain extent, maintaining BP stability (95).

Different exercise intensities differ in their effects on intestinal flora. Bernardo et al used moderate exercise intensity to study the intestinal flora of obese and hypertensive rats. Fecal samples from the rats were collected before and after exercise for 16S rRNA detection, and the results revealed that exercise changed the composition and diversity of all the intestinal microorganisms at the genus level. Streptococcus and Aggregatibacter were more abundant before exercise, whereas Allobaculum, Pseudomonas and Lactobacillus were more abundant after exercise, among which Lactobacillus was the most abundant (100). Therefore, it is not surprising that diet restriction and increased physical activity as an intervention for obese individuals resulted in a greater abundance of intestinal Bacteroides after weight loss (101). Controlling intestinal flora diversity may be another approach to reducing obesity.

There are differences in the intestinal flora between those subjected to voluntary or forced exercise, and exercise intensity may be a variable that affects the function of intestinal flora. High-intensity intermittent exercise increases the diversity of microorganisms in the distal intestine and in the feces and the ratio of Bacteroides to thick-walled flora, while also enhancing the tricarboxylic acid metabolic loop during diet-induced obesity (100). In a previous study on the effect of intestinal flora on exercise capacity, serum-free glutathione peroxidase levels in the livers of mice without specific pathogens were higher compared with those in sterile mice, and the time of endurance swimming was longer compared with that of sterile mice $(102,103)$. Other studies have demonstrated that different amounts of exercise differ in their effects, and exercise intensity may alter the composition of the intestinal flora, such that regular physical exercise exerts an anti-inflammatory effect, leading to patterns of increased anti-inflammatory cytokine and/or decreased pro-inflammatory cytokine levels $(96,104,105)$.

Exercise, an independent factor affecting the composition and diversity of intestinal flora among external environmental stressors, improves the body's metabolism and immune system. At present, there are few systematic studies on the effects of specific factors, such as exercise intensity, quantity, time and mode, on the intestinal flora. The specific regulatory mechanism of exercise on the intestinal flora awaits further study.

Effects of dietary habits on risk factors for CVD. The improvement in living standards has been accompanied by poor eating habits, such as the excessive intake of high-fat, high-cholesterol and high-salt diets, as well as tobacco and alcohol consumption, which are known causes of angiocardiopathy (106). It has been demonstrated that the benefits of a healthy diet are enhanced by the anti-inflammatory effects of SCFAs and other bioactive products produced by the intestinal flora (51). A healthy diet is conducive to the optimal functioning of the gastrointestinal tract and the composition of its microbial flora (107). Epidemiological investigations have demonstrated that Western diets are high in sugar and fat and lack sufficient dietary fiber, all of which are associated with an increased risk of obesity and CVDs (108). In addition, recent research from Tindall et al indicated that consuming FAs, bioactive compounds and the fiber in walnuts improved cardiovascular risk factors by regulating the abundance of intestinal flora (109). Moreover, it was reported that diindolylmethane and indole-3-methanol from cruciferous plants can reduce plasma TMAO levels by inhibiting flavin-containing monooxygenase, thereby preventing the development of CVDs. Therefore, the consumption of purple cabbage and broccoli may reduce TMAO levels and alleviate its adverse effects on the body (36). The evidence mentioned above supports the concept that a healthy diet containing fruits and vegetables may greatly reduce the risk factors for CVD.

\section{Conclusion}

Under normal conditions, intestinal flora is dynamically balanced to maintain health (Fig. 4). When the bodily environment, external environment and dietary factors change, causing an imbalanced flora, disease development may occur. As mentioned in this review, TMA, choline and L-carnitine are metabolites produced by intestinal flora via oxidation of FMO3 and TMAO; however, increased plasma TMA and TMAO levels exert a stimulatory effect on vascular endothelial and inflammatory cells, particularly TMAO, as it can further activate the TLR4-NLRP3-TGF- $\beta$ pathway, resulting in prominent release of inflammatory factors and collagen, eventually 


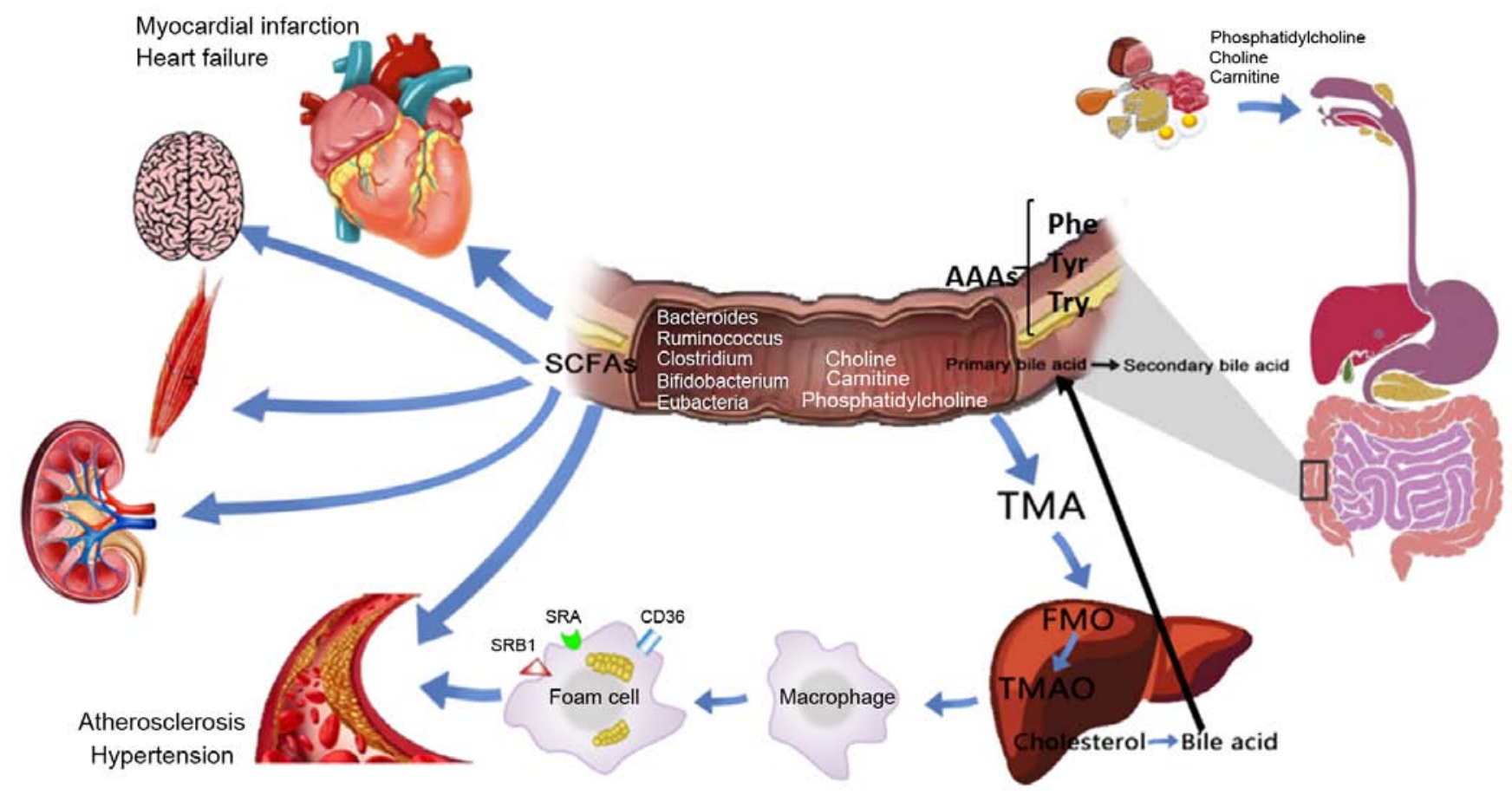

Figure 4. Potential role and mechanism of action of gut microbiota metabolites in CVD development. Dietary choline, phosphatidylcholine and carnitine produce TMA through decay and metabolism of the intestinal flora, and the absorbed TMA reaches the liver and is oxidized by FMO3 to form TMAO, which stimulates macrophages to upregulate scavenger receptors (such as CD36, SRA and SRB1), resulting in foam cell formation and accelerating the progression of chronic vascular inflammation, such as atherosclerosis. BAs enter the enterohepatic circulation through the intestinal flora and related enzymes. SCFAs are important for maintaining the normal function of the large intestine and the morphology and function of colonic epithelial cells. They can also promote the absorption of sodium and the aggregation of bacteria. Dietary proteins are metabolized by intestinal flora to produce AAAs, including Phe, Tyr and Trp, which participate in various metabolic processes. CVDs, cardiovascular diseases; TMA, trimethylamine; TMAO, trimethylamine N-oxide; FMO3, flavin monooxygenase 3; SCFAs, short-chain fatty acids; AAAs, aromatic amino acids; BAs, bile acids; Phe, phenylalanine; Trp, tryptophan; Tyr, tyrosine.

leading to myocardial fibrosis and cardiac dysfunction. TMAO promotes vascular endothelial cell dysfunction and induces atherosclerosis by activating the $\mathrm{PKC} / \mathrm{NF}-\kappa \mathrm{B} / \mathrm{VCAM}-1$ pathway. In addition, TMAO can stimulate macrophages to upregulate the CD36 and SRA scavenger receptors. When excessive lipid is deposited in the cytoplasm and exceeds the cell's own metabolic capacity, foam cells form and further accelerate the development of atherosclerosis. In addition to the inflammatory cells and cholesterol crystals present in atherosclerotic plaques, some autopsy reports show that such plaques contain bacterial DNA, making it likely that intestinal flora is translocated into the circulatory system where it participates in the disease process. BAs participate in glucose and lipid metabolism and energy consumption in the body through the enterohepatic circulation. BAs act on FXR to inhibit the abnormal proliferation of intestinal flora and to regulate the expression of BCL-2/BAX, which causes cardiomyocyte apoptosis. Through 11 $\beta$-HSD, BAs also regulate cortisol and aldosterone levels and maintain BP stability. Furthermore, BAs and their derivatives play an anti-atherosclerosis role after activating FXR. Hydrophilic BAs and the main ions inside and outside the cell membrane exert an anti-arrhythmic effect, while lipophilic BAs cause arrhythmias through changes in the membrane potential. SCFAs, which mainly comprise butyric acid, acetic acid and propionic acid, participate in the regulation of immune function by activating GPR and HDAC. The NaB metabolite from the flora activates the GPR43-Akt-GSK3 signaling pathway to regulate blood glucose homeostasis and reduce damage to the systemic blood vessels from hyperglycemia. Fecal SCFA levels in hypertensive patients are significantly higher compared with those in the plasma, suggesting that the intestinal SCFA absorption rate is low and indicating a potential target for pathogenesis and BP-lowering treatments. When AAAs undergo nitrification, halogenation, sulfonation, alkylation and acylation reactions through GPRs, they can produce substances that damage the myocardial and vascular endothelia, promoting the development of CVDs. Additionally, nitroAAAs, the metabolites formed by aromatic electrophilic substitution reactions such as acetylation, can accumulate in the body and affect the normal metabolism of cells and organ function, and the intestinal flora associated with a Trp-rich diet may produce a variety of indole metabolites, which in turn activate AhR, inhibit inflammation and reduce CVD risk factors.

In light of the current scientific knowledge and our improved understanding of intestinal flora, researchers have proposed a number of treatments that fit into what is referred to as the 'enteric-cardiac theory', such as intestinal flora transplantation, antibiotic therapy, dietary supplementation with probiotics and prebiotics, exercise and dietary adjustments. Among them, exercise and dietary changes are feasible methods for improving overall health, whereas intestinal flora transplantation may cause long-term adverse reactions. Methods for maintaining the homeostasis of the intestinal flora and improving the abundance of beneficial bacteria is a new research direction for the prevention and treatment of CVDs. Macrogenomic analysis of gut microflora in humans has revealed significant differences in the intestinal microbial structure between healthy individuals 
and patients with CVDs. The pathway of intestinal microbial metabolites may be used as an entry point for the study of treatment methods, and for in-depth research on the pathogenesis of CDVs. As the disruption of the microbial homeostatic balance is strongly associated with disease, researchers have investigated whether intestinal flora transplantation, drug interventions, dietary regulation and exercise can maintain a healthy balance of intestinal flora. Studying the pathogenesis of CVDs, such as atherosclerosis, hypertension and myocardial infarction, in the context of the microbiome may lead to the identification of new drugs and methods with few side effects, cost-effectiveness and good curative efficacy.

\section{Acknowledgements}

Not applicable.

\section{Funding}

The present study was supported by National Natural Science Foundation of China (grant nos. 81970056, 81700269 and 81741129), Southern Marine Science and Engineering Guangdong Laboratory Zhanjiang (grant no.ZJW-2019-007), Natural Science Foundation of Guangdong Province (grant no. 2019A1515011925), and Science and technology plan project of Zhanjiang (grant no. 2019A01002).

\section{Availability of data and materials}

Not applicable.

\section{Authors' contributions}

WL and HL contributed to the conception and design of the study. YZ, XS and ZH performed the literature searches and wrote the manuscript. YZ prepared the figures. WL, ZL, YH, YQ and CC wrote and revised the manuscript. All the authors have read and approved the final manuscript.

\section{Ethics approval and consent to participate}

Not applicable.

\section{Patient consent for publication}

Not applicable.

\section{Competing interests}

The authors declare that they have no competing interests.

\section{References}

1. GBD 2015 Mortality and Causes of Death Collaborators: Global, regional, and national life expectancy, all-cause mortality, and cause-specific mortality for 249 causes of death, 1980-2015: A systematic analysis for the global burden of disease study 2015 . Lancet 388: 1459-1544, 2016.

2. Drouin N, Kloots T, Schappler J, Rudaz S, Kohler I, Harms A, Lindenburg PW and Hankemeier T: Electromembrane extraction of highly polar compounds: Analysis of cardiovascular biomarkers in plasma. Metabolites 10: 4, 2019.
3. Andersson $\mathrm{C}$ and Vasan RS: Epidemiology of cardiovascular disease in young individuals. Nat Rev Cardiol 15: 230-240, 2018.

4. Maqbool M, Cooper ME and Jandeleit-Dahm KAM: Cardiovascular disease and diabetic kidney disease. Semin Nephrol 38: 217-232, 2018.

5. Defesche JC, Gidding SS, Harada-Shiba M, Hegele RA, Santos RD and Wierzbicki AS: Familial hypercholesterolaemia. Nat Rev Dis Primers 3: 17093, 2017.

6. Jabbar A, Pingitore A, Pearce SH, Zaman A, Iervasi G and Razvi S: Thyroid hormones and cardiovascular disease. Nat Rev Cardiol 14: 39-55, 2017.

7. Backhed F, Ding H, Wang T, Hooper LV, Koh GY, Nagy A, Semenkovich CF and Gordon JI: The gut microbiota as an environmental factor that regulates fat storage. Proc Natl Acad Sci USA 101: 15718-15723, 2004.

8. Zhu W, Gregory JC, Org E, Buffa JA, Gupta N, Wang Z, Li L, Fu X, Wu Y, Mehrabian M, et al: Gut microbial metabolite TMAO enhances platelet hyperreactivity and thrombosis risk. Cell 165: 111-124, 2016.

9. Perez NB, Dorsen C and Squires A: Dysbiosis of the gut microbiome: A concept analysis. J Holist Nurs 898010119879527, 2019 (Epub ahead of print).

10. Skye SM, Zhu W, Romano KA, Guo CJ, Wang Z, Jia X, Kirsop J, Haag B, Lang JM, DiDonato JA, et al: Microbial transplantation with human gut commensals containing CutCis sufficient to transmit enhanced platelet reactivity and thrombosis potential. Circ Res 123: 1164-1176, 2018.

11. Fu J and Kuipers F: Systems genetics approach reveals cross-talk between bile acids and intestinal microbes. PLoS Genet 15: e1008307, 2019.

12. Ohira H, Tsutsui W and Fujioka Y: Are short chain fatty acids in gut microbiota defensive players for inflammation and atherosclerosis? J Atheroscler Thromb 24: 660-672, 2017.

13. Devlin AS, Marcobal A, Dodd D, Nayfach S, Plummer N, MeyerT, Pollard KS, Sonnenburg JL and Fischbach MA: Modulation of a circulating uremic solute via rational genetic manipulation of the gut microbiota. Cell Host Microbe 20: 709-715, 2016.

14. Yao C, Chen BH, Joehanes R, Otlu B, Zhang X, Liu C, Huan T, Tastan O, Cupples LA, Meigs JB, et al: Integromic analysis of genetic variation and gene expression identifies networks for cardiovascular disease phenotypes. Circulation 131: 536-549, 2015.

15. Zhang WQ, Zhao TT, Gui DK, Gao CL, Gu JL, Gan WJ, Huang W, $\mathrm{Xu} \mathrm{Y}, \mathrm{Zhou} \mathrm{H}$, Chen WN, et al: Sodium butyrate improves liver glycogen metabolism in Type 2 diabetes mellitus. J Agric Food Chem 67: 7694-7705, 2019.

16. Odegaard AO, Koh WP, Gross MD, Yuan JM and Pereira MA: Combined lifestyle factors and cardiovascular disease mortality in Chinese men and women: The Singapore Chinese health study. Circulation 124: 2847-2854, 2011.

17. Macfarlane GT and Macfarlane S: Bacteria, colonic fermentation, and gastrointestinal health. J AOAC Int 95: 50-60, 2012

18. Tang WH, Kitai T and Hazen SL: Gut microbiota in cardiovascular health and disease. Circ Res 120: 1183-1196, 2017.

19. Lam V, Su J, Hsu A, Gross GJ, Salzman NH and Baker JE: Intestinal microbial metabolites are linked to severity of myocardial infarction in rats. PLoS One 11: e0160840, 2016.

20. Caro-Gomez E, Sierra JA, Escobar JS, Alvarez-Quintero R, Naranjo M, Medina S, Velasquez-Mejia EP, Tabares-Guevara JH, Jaramillo JC, Leon-Varela YM, et al: Green coffee extract improves cardiometabolic parameters and modulates gut microbiota in High-Fat-Diet-Fed ApoE ${ }^{-/-}$Mice. Nutrients 11: 497, 2019.

21. Tran HQ,Ley RE, Gewirtz AT and Chassaing B: Flagellin-elicited adaptive immunity suppresses flagellated microbiota and vaccinates against chronic inflammatory diseases. Nat Commun 10: $5650,2019$.

22. Wang Z, Roberts AB, Buffa JA, Levison BS, Zhu W, Org E, Gu X, Huang Y, Zamanian-Daryoush M, Culley MK, et al: Non-lethal inhibition of gut microbial trimethylamine production for the treatment of atherosclerosis. Cell 163: 1585-1595, 2015.

23. Grabherr F, Grander C, Effenberger M, Adolph TE and Tilg H: Gut dysfunction and non-alcoholic fatty liver disease. Front Endocrinol (Lausanne) 10: 611, 2019.

24. Ramirez-Perez O, Cruz-Ramon V, Chinchilla-Lopez P and Mendez-Sanchez N: The role of the gut microbiota in bile acid metabolism. Ann Hepato 16 (Suppl. 1: S3-105.): S21-S20, 2017.

25. Chen H, Peng L, Perez de Nanclares M, Trudeau MP, Yao D, Cheng Z, Urriola PE, Mydland LT, Shurson GC, Overland M and Chen $C$ : Identification of sinapine-derived choline from a rapeseed diet as a source of serum Trimethylamine $\mathrm{N}$-Oxide in pigs. J Agric Food Chem 67: 7748-7754, 2019. 
26. Wang Z, Klipfell E, Bennett BJ, Koeth R, Levison BS, Dugar B, Feldstein AE, Britt EB, Fu X, Chung YM, et al: Gut flora metabolism of phosphatidylcholine promotes cardiovascular disease. Nature 472: 57-63, 2011.

27. Ufnal M, Zadlo A and Ostaszewski R: TMAO: A small molecule of great expectations. Nutrition 31: 1317-1323, 2015.

28. Koeth RA, Wang Z, Levison BS, Buffa JA, Org E, Sheehy BT, Britt EB, Fu X, Wu Y,Li L, et al: Intestinal microbiota metabolism of L-carnitine, a nutrient in red meat, promotes atherosclerosis. Nat Med 19: 576-585, 2013

29. Suzuki H, Kurihara Y, Takeya M, Kamada N, Kataoka M, Jishage K, Ueda O, Sakaguchi H, Higashi T, Suzuki T, et al: A role for macrophage scavenger receptors in atherosclerosis and susceptibility to infection. Nature 386: 292-296, 1997.

30. Brown JM and Hazen SL: The gut microbial endocrine organ: Bacterially derived signals driving cardiometabolic diseases. Annu Rev Med 66: 343-359, 2015.

31. Li XS, Obeid S, Klingenberg R, Gencer B, Mach F, Raber L, Windecker S, Rodondi N, Nanchen D, Muller O, et al: Gut microbiota-dependent trimethylamine $\mathrm{N}$-oxide in acute coronary syndromes: A prognostic marker for incident cardiovascular events beyond traditional risk factors. Eur Heart J 38: 814-824, 2017.

32. Liu TX, Niu HT and Zhang SY: Intestinal microbiota metabolism and atherosclerosis. Chin Med J (Engl) 128: 2805-2811, 2015.

33. Ott SJ, El Mokhtari NE, Musfeldt M, Hellmig S, Freitag S, Rehman A, Kuhbacher T, Nikolaus S, Namsolleck P, Blaut M, et al: Detection of diverse bacterial signatures in atherosclerotic lesions of patients with coronary heart disease. Circulation 113: 929-937, 2006

34. Winther SA, Ollgaard JC, Tofte N, Tarnow L, Wang Z, Ahluwalia TS, Jorsal A, Theilade S, Parving HH,Hansen TW, et al: Utility of plasma concentration of Trimethylamine N-Oxide in predicting cardiovascular and renal complications in individuals with type 1 diabetes. Diabetes Care 42: 1512-1520, 2019.

35. Li X, Geng J, Zhao J, Ni Q, Zhao C, Zheng Y, Chen X and Wang L: Trimethylamine N-Oxide exacerbates cardiac fibrosis via activating the NLRP3 inflammasome. Front Physiol 10: 866, 2019.

36. Shepshelovich J, Goldstein-Magal L, Globerson A, Yen PM, Rotman-Pikielny P and Hirschberg K: Protein synthesis inhibitors and the chemical chaperone TMAO reverse endoplasmic reticulum perturbation induced by overexpression of the iodide transporter pendrin. J Cell Sci 118 (Pt 8): 1577-1586, 2005.

37. Chen S, Henderson A, Petriello MC, Romano KA, Gearing M, Miao J, Schell M, Sandoval-Espinola WJ, Tao J, Sha B, et al Trimethylamine N-Oxide binds and activates PERK to promote metabolic dysfunction. Cell Metab 30: 1141-1151 e5, 2019.

38. Ma G, Pan B, Chen Y, Guo C, Zhao M, Zheng L and Chen B Trimethylamine N-oxide in atherogenesis: Impairing endothelial self-repair capacity and enhancing monocyte adhesion. Biosci Rep 37: BSR20160244, 2017.

39. Shiffka SJ, Kane MA and Swaan PW: Planar bile acids in health and disease. Biochim Biophys Acta Biomembr 1859: 2269-2276, 2017.

40. Chiang JY: Bile acid regulation of gene expression: Roles of nuclear hormone receptors. Endocr Rev 23: 443-463, 2002

41. Lefebvre P, Cariou B, Lien F, Kuipers F and Staels B: Role of bile acids and bile acid receptors in metabolic regulation. Physiol Rev 89: 147-191, 2009.

42. Hoving LR, Katiraei S, Heijink M, Pronk A, van der Wee-Pals L, Streefland T, Giera M, Willems van Dijk K and van Harmelen V: Dietary mannan oligosaccharides modulate gut microbiota, increase fecal bile acid excretion, and decrease plasma cholesterol and atherosclerosis development. Mol Nutr Food Res 62: e1700942, 2018

43. Sun L, Pang Y, Wang X, Wu Q, Liu H, Liu B, Liu G, Ye M, Kong $\mathrm{W}$ and Jiang C: Ablation of gut microbiota alleviates obesity-induced hepatic steatosis and glucose intolerance by modulating bile acid metabolism in hamsters. Acta Pharm Sin B 9: 702-710, 2019.

44. Tsuei J, Chau T, Mills D and Wan YJ: Bile acid dysregulation, gut dysbiosis, and gastrointestinal cancer. Exp Biol Med (Maywood) 239: 1489-1504, 2014.

45. Dodd D, Spitzer MH, Van Treuren W, Merrill BD Hryckowian AJ, Higginbottom SK, Le A, Cowan TM, Nolan GP, Fischbach MA and Sonnenburg JL: A gut bacterial pathway metabolizes aromatic amino acids into nine circulating metabolites. Nature 551: 648-652, 2017.

46. Jia ET, Liu ZY, Pan M, Lu JF and Ge QY: Regulation of bile acid metabolism-related signaling pathways by gut microbiota in diseases. J Zhejiang Univ Sci B 20: 781-792, 2019.
47. Merritt ME and Donaldson JR: Effect of bile salts on the DNA and membrane integrity of enteric bacteria. J Med Microbiol 58 (Pt 12): 1533-1541, 2009.

48. Lin S, Yang X, Yuan P, Yang J, Wang P, Zhong H, Zhang X, Che L, Feng B, Li J, et al: Undernutrition shapes the gut microbiota and bile acid profile in association with altered gut-liver FXR signaling in weaning pigs. J Agric Food Chem 67: 3691-3701, 2019.

49. Wu P, Zhang Y, Liu Y, Wang X, Guo Z, Zhang Y, Liang X and Lai W: Effects of cholic acid on blood pressure and production of vascular aldosterone and corticosterone. Steroids 64: 291-295, 1999.

50. Valdivia C, Carvajal CA, Campino C, Allende F, Martinez-Aguayo A, Baudrand R, Vecchiola A, Lagos CF, Tapia-Castillo A, Fuentes CA, et al: Citosine-adenine-repeat microsatellite of $11 \beta$-hydroxysteroid dehydrogenase 2 gene in hypertensive children. Am J Hypertens 29: 25-32, 2016.

51. Wan Y, Wang F, Yuan J, Li J, Jiang D, Zhang J, Li H, Wang R, Tang J, Huang T, et al: Effects of dietary fat on gut microbiota and faecal metabolites, and their relationship with cardiometabolic risk factors: A 6-month randomised controlled-feeding trial. Gut 68: 1417-1429, 2019.

52. Charach G, Karniel E, Novikov I, Galin L, Vons S, Grosskopf I and Charach L: Reduced bile acid excretion is an independent risk factor for stroke and mortality: A prospective follow-up study. Atherosclerosis 293: 79-85, 2020.

53. Fedorova OV, Zernetkina VI, Shilova VY, Grigorova YN, Juhasz O, Wei W, Marshall CA, Lakatta EG and Bagrov AY: Synthesis of an Endogenous steroidal Na Pump inhibitor marinobufagenin, implicated in human cardiovascular diseases, is initiated by CYP27A1 via Bile Acid pathway. Circ Cardiovasc Genet 8: 736-745, 2015.

54. Rainer PP, Primessnig U, Harenkamp S, Doleschal B, Wallner M, Fauler G, Stojakovic T, Wachter R, Yates A, Groschner K, et al: Bile acids induce arrhythmias in human atrial myocardium-implications for altered serum bile acid composition in patients with atrial fibrillation. Heart 99: 1685-1692, 2013.

55. Pu J, Yuan A, Shan P, Gao E, Wang X, Wang Y, Lau WB, Koch W, Ma XL and He B: Cardiomyocyte-expressed farnesoid-X-receptor is a novel apoptosis mediator and contributes to myocardial ischaemia/reperfusion injury. Eur Heart J 34: 1834-1845, 2013

56. Chen ML, Yi L, Zhang Y, Zhou X, Ran L, Yang JN, Zhu JD, Zhang QY and Mi MT: Resveratrol attenuates Trimethylamine-N-Oxide (TMAO)-Induced atherosclerosis by regulating TMAO Synthesis and bile acid metabolism via remodeling of the Gut microbiota. mBio 7: e02210-15, 2016.

57. Nguyen MT, Favelyukis S, Nguyen AK, Reichart D, Scott PA, Jenn A, Liu-Bryan R, Glass CK, Neels JG and Olefsky JM: A subpopulation of macrophages infiltrates hypertrophic adipose tissue and is activated by free fatty acids via Toll-like receptors 2 and 4 and JNK-dependent pathways. J Biol Chem 282: 35279-35292, 2007.

58. Calderon-Perez L, Gosalbes MJ, Yuste S, Valls RM, Pedret A, Llaurado E, Jimenez-Hernandez N, Artacho A, Pla-Paga L, Companys J, et al: Gut metagenomic and short chain fatty acids signature in hypertension: A cross-sectional study. Sci Rep 10: 6436, 2020.

59. Morrison DJ and Preston T: Formation of short chain fatty acids by the gut microbiota and their impact on human metabolism. Gut Microbes 7: 189-200, 2016.

60. Manrique Vergara D and González Sánchez ME: Short chain fatty acids (butyric acid) and intestinal diseases. Nutr Hosp 34 (Suppl 4): S58-S61, 2017 (In Spanish).

61. Sun M, Wu W, Liu Z and Cong Y: Microbiota metabolite short chain fatty acids, GPCR, and inflammatory bowel diseases. J Gastroenterol 52: 1-8, 2017.

62. Smith PM, Howitt MR, Panikov N, Michaud M, Gallini CA, Bohlooly-Y M, Glickman JN and Garrett WS: The microbial metabolites, short-chain fatty acids, regulate colonic Treg cell homeostasis. Science 341: 569-573, 2013.

63. Li J, Zhao F, Wang Y, Chen J, Tao J, Tian G, Wu S, Liu W, Cui Q, Geng B, et al: Gut microbiota dysbiosis contributes to the development of hypertension. Microbiome 5: 14, 2017.

64. Le B, Bůžková P, Robbins JA, Fink HA, Raiford M, Isales CM, Shikany JM, Coughlin SS and Carbone LD: The association of aromatic amino acids with incident hip fracture, aBMD, and body composition from the cardiovascular health study. Calcif Tissue Int 105: 161-172, 2019. 
65. Tessari P, Lante A and Mosca G: Essential amino acids: Master regulators of nutrition and environmental footprint? Sci Rep 6: 26074, 2016.

66. Fernstrom JD and Fernstrom MH: Tyrosine, phenylalanine, and catecholamine synthesis and function in the brain. J Nutr 137 (6 Suppl 1): 1539S-1547S; discussion 1548S, 2007.

67. Shishehbor MH, Aviles RJ, Brennan ML, Fu X, Goormastic M, Pearce GL, Gokce N, Keaney JF Jr, Penn MS, Sprecher DL, et al: Association of nitrotyrosine levels with cardiovascular disease and modulation by statin therapy. JAMA 289: 1675-1680, 2003.

68. Thomson L: 3-nitrotyrosine modified proteins in atherosclerosis. Dis Markers 2015: 708282, 2015.

69. Haase S, Haghikia A, Wilck N, Müller DN and Linker RA: Impacts of microbiome metabolites on immune regulation and autoimmunity. Immunology 154: 230-238, 2018.

70. Brawner KM, Yeramilli VA, Duck LW, Van Der Pol W, Smythies LE, Morrow CD, Elson CO and Martin CA: Depletion of dietary aryl hydrocarbon receptor ligands alters microbiota composition and function. Sci Rep 9: 14724, 2019.

71. Zelante T, Iannitti RG, Cunha C, De Luca A, Giovannini G, Pieraccini G, Zecchi R, D'Angelo C, Massi-Benedetti C, Fallarino F, et al: Tryptophan catabolites from microbiota engage aryl hydrocarbon receptor and balance mucosal reactivity via interleukin-22. Immunity 39: 372-385, 2013.

72. Metghalchi S, Ponnuswamy P, Simon T, Haddad Y, Laurans L, Clément M, Dalloz M, Romain M, Esposito B, Koropoulis V, et al: Indoleamine 2,3-dioxygenase fine-tunes immune homeostasis in atherosclerosis and colitis through repression of interleukin-10 production. Cell Metab 22: 460-471, 2015.

73. Laurans L, Venteclef N, Haddad Y, Chajadine M, Alzaid F Metghalchi S, Sovran B, Denis RGP, Dairou J, Cardellini M, et al: Genetic deficiency of indoleamine 2,3-dioxygenase promotes gut microbiota-mediated metabolic health. Nat Med 24: 1113-1120, 2018.

74. Lamas B, Richard ML, Leducq V, Pham HP, Michel ML, Da Costa G, Bridonneau C, Jegou S, Hoffmann TW, Natividad JM, et al: CARD9 impacts colitis by altering gut microbiota metabolism of tryptophan into aryl hydrocarbon receptor ligands. Nat Med 22: 598-605, 2016

75. Hoyles L, Fernández-Real JM, Federici M, Serino M, Abbott J, Charpentier J, Heymes C, Luque JL, Anthony E, Barton RH, et al: Molecular phenomics and metagenomics of hepatic steatosis in non-diabetic obese women. Nat Med 24: 1070-1080, 2018.

76. Kakihana K, Fujioka Y, Suda W, Najima Y, Kuwata G, Sasajima S Mimura I, Morita H, Sugiyama D, Nishikawa H, et al: Fecal microbiota transplantation for patients with steroid-resistant acute graft-versus-host disease of the gut. Blood 128: 2083-2088, 2016.

77. Moludi J, Maleki V, Jafari-Vayghyan H, Vaghef-Mehrabany E and Alizadeh M: Metabolic endotoxemia and cardiovascular disease: A systematic review about potential roles of prebiotics and probiotics. Clin Exp Pharmacol Physiol 47: 927-939, 2020.

78. Gregory JC, Buffa JA, Org E, Wang Z, Levison BS, Zhu W, Wagner MA, Bennett BJ, Li L, DiDonato JA, et al: Transmission of atherosclerosis susceptibility with gut microbial transplantation. J Biol Chem 290: 5647-5660, 2015.

79. Lu F, Liu F, Zhou Q, Hu X and Zhang Y: Effects of grape pomace and seed polyphenol extracts on the recovery of gut microbiota after antibiotic treatment in high-fat diet-fed mice. Food Sci Nutr 7: 2897-2906, 2019.

80. Liao X, Song L, Zeng B, Liu B, Qiu Y, Qu H, Zheng Y, Long M, Zhou H, Wang Y, et al: Alteration of gut microbiota induced by DPP-4i treatment improves glucose homeostasis. EBioMedicine 44: 665-674, 2019.

81. Wan JJ, Lin CH, Ren ED, Su Y and Zhu WY: Effects of early intervention with maternal fecal bacteria and antibiotics on liver metabolome and transcription in neonatal pigs. Front Physiol 10: $171,2019$.

82. Janeiro MH, Ramirez MJ, Milagro FI, Martinez JA and Solas M: Implication of trimethylamine N-Oxide (TMAO) in disease: Potential biomarker or new therapeutic target. Nutrients 10 : 1398, 2018

83. Queipo-Ortuño MI, Seoane LM, Murri M, Pardo M, Gomez-Zumaquero JM, Cardona F, Casanueva F and Tinahones FJ: Gut microbiota composition in male rat models under different nutritional status and physical activity and its association with serum leptin and ghrelin levels. PLoS One 8 e65465, 2013.

84. Le Roy T, Lécuyer E, Chassaing B, Rhimi M, Lhomme M, Boudebbouze S, Ichou F, Haro Barceló J, Huby T, Guerin M, et al: The intestinal microbiota regulates host cholesterol homeostasis. BMC Biol 17: 94, 2019.
85. Heymsfield SB and Wadden TA: Mechanisms, pathophysiology, and management of obesity. N Engl J Med 376: 254-266, 2017.

86. Guirro M, Costa A, Gual-Grau A, Herrero P, Torrell H, Canela N and Arola L: Effects from diet-induced gut microbiota dysbiosis and obesity can be ameliorated by fecal microbiota transplantation: A multiomics approach. PLoS One 14: e0218143, 2019.

87. Enright EF, Joyce SA, Gahan CG and Griffin BT: Impact of gut microbiota-mediated bile acid metabolism on the solubilization capacity of bile salt micelles and drug solubility. Mol Pharm 14: 1251-1263, 2017.

88. Langlands SJ, Hopkins MJ, Coleman N and Cummings JH: Prebiotic carbohydrates modify the mucosa associated microflora of the human large bowel. Gut 53: 1610-1616, 2004

89. Hill C, Guarner F, Reid G, Gibson GR, Merenstein DJ, Pot B Morelli L, Canani RB, Flint HJ, Salminen S, et al: Expert consensus document. The International Scientific Association for Probiotics and Prebiotics consensus statement on the scope and appropriate use of the term probiotic. Nat Rev Gastroenterol Hepatol 11: 506-514, 2014.

90. Gallo A, Passaro G, Gasbarrini A, Landolfi R and Montalto M: Modulation of microbiota as treatment for intestinal inflammatory disorders: An uptodate. World J Gastroenterol 22: 7186-7202, 2016.

91. Quigley EM: Prebiotics and probiotics: Their role in the management of gastrointestinal disorders in adults. Nutr Clin Pract 27: 195-200, 2012.

92. Mozaffarian D: Dairy foods, obesity, and metabolic health: The role of the food matrix compared with single nutrients. Adv Nutr 10: 917S-923S, 2019.

93. Chen Q, Liu M, Zhang P, Fan S, Huang J, Yu S, Zhang C and $\mathrm{Li} \mathrm{H}$ : Fucoidan and galactooligosaccharides ameliorate high-fat diet-induced dyslipidemia in rats by modulating the gut microbiota and bile acid metabolism. Nutrition 65: 50-59, 2019.

94.Francavilla G, Abrignani MG, Braschi A, Sciacca R, Francavilla VC, Caracciolo MM, Renda N, Riccio C, Scaglione A and Braschi G: Physical exercise and sport activities in patients with and without coronary heart disease. Monaldi Arch Chest Dis 68: 87-95, 2007 (In Italian)

95. Cheng YJ, Zhao XJ, Zeng W, Xu MC, Ma YC and Wang M: Effect of intradialytic exercise on physical performance and cardiovascular risk factors in patients receiving maintenance hemodialysis: A pilot and feasibility study. Blood Purif 1-10, 2019 (Epub ahead of print).

96. Codella R, Luzi L and Terruzzi I: Exercise has the guts: How physical activity may positively modulate gut microbiota in chronic and immune-based diseases. Dig Liver Dis 50: 331-341, 2018.

97. Chen J, Guo Y, Gui Y and Xu D: Physical exercise, gut, gut microbiota, and atherosclerotic cardiovascular diseases. Lipids Health Dis 17: 17, 2018

98. Ito S: High-intensity interval training for health benefits and care of cardiac diseases-The key to an efficient exercise protocol. World J Cardiol 11: 171-188, 2019.

99. Kang Y and Cai Y: Gut microbiota and hypertension: From pathogenesis to new therapeutic strategies. Clin Res Hepatol Gastroenterol 42: 110-117, 2018.

100. Petriz BA, Castro AP, Almeida JA, Gomes CP, Fernandes GR, Kruger RH, Pereira RW and Franco OL: Exercise induction of gut microbiota modifications in obese, non-obese and hypertensive rats. BMC Genomics 15: 511, 2014

101. Verdam FJ, Fuentes S, de Jonge C, Zoetendal EG, Erbil R, Greve JW, Buurman WA, de Vos WM and Rensen SS: Human intestinal microbiota composition is associated with local and systemic inflammation in obesity. Obesity (Silver Spring) 21: E607-E615, 2013

102. Denou E, Marcinko K, Surette MG, Steinberg GR and Schertzer JD: High-intensity exercise training increases the diversity and metabolic capacity of the mouse distal gut microbiota during diet-induced obesity. Am J Physiol Endocrinol Metab 310: E982-E993, 2016.

103. Hsu YJ, Chiu CC, Li YP, Huang WC, Huang YT, Huang CC and Chuang HL: Effect of intestinal microbiota on exercise performance in mice. J Strength Cond Res 29: 552-558, 2015.

104. Starkie R, Ostrowski SR, Jauffred S, Febbraio $M$ and Pedersen BK: Exercise and IL-6 infusion inhibit endotoxin-induced TNF-alpha production in humans. FASEB J 17: 884-886, 2003.

105. Pedersen BK and Saltin B: Exercise as medicine-evidence for prescribing exercise as therapy in 26 different chronic diseases. Scand J Med Sci Sports 25 (Suppl 3): S1-S72, 2015. 
106. Fung TT, Rexrode KM, Mantzoros CS, Manson JE, Willett WC and Hu FB: Mediterranean diet and incidence of and mortality from coronary heart disease and stroke in women. Circulation 119: 1093-1100, 2009.

107. Holscher HD, Guetterman HM, Swanson KS, An R, Matthan NR, Lichtenstein AH, Novotny JA and Baer DJ: Walnut consumption alters the gastrointestinal microbiota, microbially derived secondary bile acids, and health markers in healthy adults: A randomized controlled trial. J Nutr 148: 861-867, 2018.

108. Psaltopoulou T, Hatzis G, Papageorgiou N, Androulakis E, Briasoulis A and Tousoulis D: Socioeconomic status and risk factors for cardiovascular disease: Impact of dietary mediators. Hellenic J Cardiol 58: 32-42, 2017.
109. Tindall AM, Mclimans CJ, Petersen KS, Kris-Etherton PM and Lamendella R: Walnuts and vegetable oils containing oleic acid differentially affect the gut microbiota and associations with cardiovascular risk factors: Follow-up of a randomized, controlled, feeding trial in adults at risk for cardiovascular disease. J Nutr 150: 806-817, 2020.

(i) (9) This work is licensed under a Creative Commons Attribution-NonCommercial-NoDerivatives 4.0 International (CC BY-NC-ND 4.0) License. 